Article

\title{
Novel Bioactive Penicipyrroether A and Pyrrospirone J from the Marine-Derived Penicillium sp. ZZ380
}

\author{
Tengfei Song ${ }^{2} \mathbb{D}$, Mingmin Tang ${ }^{1}$, Hengju Ge ${ }^{2}$, Mengxuan Chen ${ }^{2}$, Xiaoyuan Lian ${ }^{1, *}$ and \\ Zhizhen Zhang $2, *$ (D) \\ 1 College of Pharmaceutical Sciences, Zhejiang University, Hangzhou 310058, China; tmm0907@163.com \\ 2 Ocean College, Zhoushan Campus, Zhejiang University, Zhoushan 316021, China; \\ sdasheng72@163.com (T.S.); 15805152141@163.com (H.G.); zerocmx@163.com (M.C.) \\ * Correspondence: xylian@zju.edu.cn (X.L.); zzhang88@zju.edu.cn (Z.Z.); \\ Tel.: +86-135-7547-6388 (X.L.); +86-136-7585-9706 (Z.Z.)
}

Received: 9 April 2019; Accepted: 7 May 2019; Published: 15 May 2019

check for updates

\begin{abstract}
The marine-sourced fungus Penicillium sp. ZZ380 was previously reported to have the ability to produce a series of new pyrrospirone alkaloids. Further investigation on this strain resulted in the isolation and identification of novel penicipyrroether A and pyrrospirone J. Each of them represents the first example of its structural type, with a unique 6/5/6/5 polycyclic fusion that is different from the 6/5/6/6 fused ring system for the reported pyrrospirones. Their structures were elucidated by extensive nuclear magnetic resonance (NMR) and high resolution electrospray ionization mass spectroscopy (HRESIMS) spectroscopic analyses, electronic circular dichroism (ECD) and ${ }^{13} \mathrm{C}$ NMR calculations and X-ray single crystal diffraction. Penicipyrroether A showed potent antiproliferative activity against human glioma U87MG and U251 cells with half maximal inhibitory concentration $\left(\mathrm{IC}_{50}\right)$ values of $1.64-5.50 \mu \mathrm{M}$ and antibacterial inhibitory activity with minimum inhibitory concentration (MIC) values of $1.7 \mu \mathrm{g} / \mathrm{mL}$ against methicillin-resistant Staphylococcus aureus and $3.0 \mu \mathrm{g} / \mathrm{mL}$ against Escherichia coli.
\end{abstract}

Keywords: marine fungus; Penicillium sp. ZZ380; penicipyrroether A; pyrrospirone J; antiproliferative and antibacterial activities

\section{Introduction}

Hirsutellones and related compounds are a family of fungal secondary metabolites that bear a unique 13-membered ether ring, which is composed of the structural units of decahydrofluorene, para-cyclophane and pyrrolidinone [1,2]. This family of fungal metabolites is divided into four groups: GKK1032s, hirsutellones, pyrrocidines and pyrrospirones. To date, about 29 such metabolites have been isolated and identified from fungal sources of genera Cylindrocarpon, Embellisia, Hirsutella, Lewia, Neonectria, Penicillium and Trichoderma and some of these metabolites have proved to have cytotoxic, antifungal and antibacterial activities [1-4].

A marine fungus Penicillium sp. ZZ380 [2] was recently isolated from a wild sea crab Pachygrapsus crassipes during the course of our ongoing program to discover novel bioactive agents from marine microorganisms. This fungus in the BMPM liquid medium produced new pyrrospirones C-I (1-7) [2], penicipyrrodiether A (8) [3] and compound 10 (Figure 1), the structure of which was not possible to establish previously, due to its structural instability. Pyrrospirone G (5) was found to have potent activity against the proliferation of different glioma cells and pyrrospirones C (1), F (4), I (7) and penicipyrrodiether A (8) showed activities in inhibiting the growth of methicillin-resistant Staphylococcus aureus (MRSA) and Escherichia coli. 


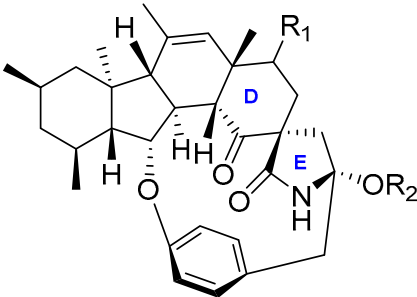

1: $\mathrm{R}_{1}=\alpha \mathrm{OH}, \mathrm{R}_{2}=\mathrm{CH}_{3}$

2: $\mathrm{R}_{1}=\beta \mathrm{OH}, \mathrm{R}_{2}=\mathrm{CH}_{3}$

3: $\mathrm{R}_{1}=\alpha \mathrm{OH}, \mathrm{R}_{2}=\mathrm{H}$

4: $R_{1}=\beta O H, R_{2}=H$

5: $\mathrm{R}_{1}=\mathrm{O}, \mathrm{R}_{2}=\mathrm{H}$

6: $R_{1}=H, R_{2}=H$

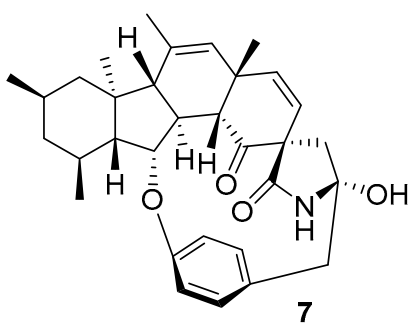

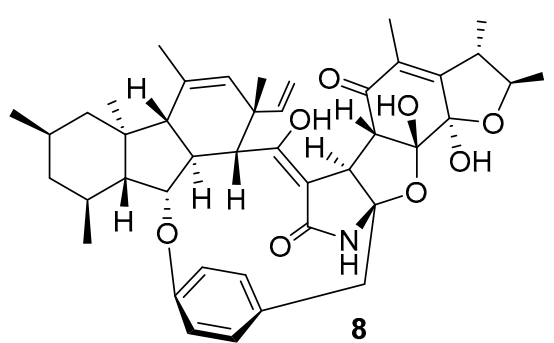

8<smiles>Cc1cc(O)c(C)c(O)c1C</smiles><smiles>CO[C@H]1CCc2oc3cc(C)cc(O)c3c(=O)c21</smiles>

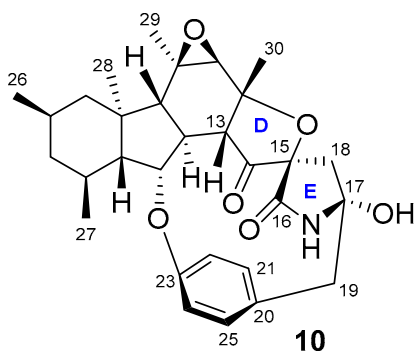

10<smiles>COC(=O)c1c(O)ccc2oc3cc(C)cc(O)c3c(=O)c12</smiles><smiles></smiles>

16<smiles>Cc1c(C)c2c3c(c1O)OC31O[C@H](C)C(C)[C@@H](C)c3c(C)c(O)cc(c31)O2</smiles>

17

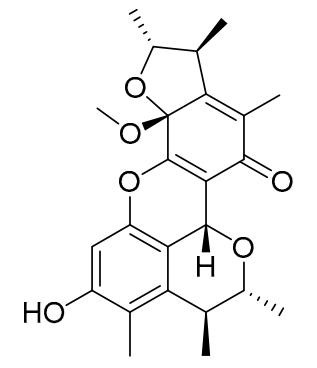

18

Figure 1. Structures of compounds 1-18 isolated from the cultures of Penicillium sp. ZZ380.

It is well known that OSMAC (One Strain, Many Compounds) has been used to activate the silent gene cluster of microbial secondary metabolic biosynthesis to express and then produce different novel bioactive natural products [5-7]. In order to obtain more bioactive compounds from this pyrrospirones-produced fungus ZZ380, a different liquid medium of PDB (potato dextrose broth) was used to culture strain ZZ380 in this study, resulting in the isolation of nine compounds, including novel penicipyrroether A (9) (Figure 1) but no pyrrospirones and penicipyrrodiether A. Herein, we report the culture of strain ZZ380 in PDB medium as well as the structural determination and bioactive evaluation of penicipyrroether A (9) and the previously unidentified compound $\mathbf{1 0 .}$

\section{Results and Discussion}

An EtOAc extract prepared from a mass culture of Penicillium sp. ZZ380 in PDB medium was separated by ODS column chromatography, followed by HPLC purification, to give compounds 9 and 11-18. Compound 10 was isolated from a previous culture of ZZ380 in BMPM medium [2]. On the basis of the nuclear magnetic resonance (NMR) data and specific rotation, as well as the comparison with the reported data, eight known compounds were identified as 2,4,5-trimethylresorcinol (11) [8], stoloniferol B (12) [9], coniochaetone E (13) [10], pinselin (14) [11], quinolactacin $A_{1}$ (15) [12], ergosterol (16) [13], penicitrinol A (17) [14] and B (18) [15]. Their ${ }^{13} \mathrm{C}$ NMR data are presented in Tables S1 and S2 (Supplementary Materials). 
Compound 9 was obtained as colorless block crystals from $\mathrm{MeOH}$ and has a molecular formula $\mathrm{C}_{32} \mathrm{H}_{41} \mathrm{NO}_{5}$ deduced from its high resolution electrospray ionization mass spectroscopy (HRESIMS) ions at $m / z[\mathrm{M}+\mathrm{H}]^{+} 520.3063$ and $[\mathrm{M}+\mathrm{Na}]^{+} 542.2877$ as well as its ${ }^{13} \mathrm{C} \mathrm{NMR}$ data. Analyses of its ${ }^{1} \mathrm{H},{ }^{13} \mathrm{C},{ }^{1} \mathrm{H}-{ }^{1} \mathrm{H}$ COSY, HSQC and HMBC spectra (Figures S1-S17) indicated that the structural parts of rings $\mathrm{A}-\mathrm{C}, \mathrm{F}$ and $\mathrm{G}$ for 9 and pyrrospirones $\mathrm{C}-\mathrm{I}(\mathbf{1 - 7 )}$ are the same. However, the structures of 9 and pyrrospirones have significant differences. The first difference is the ring $\mathrm{D}$ with a five-membered ether ring for $\mathbf{9}$ and a cyclohexanone for pyrrospirones. The second difference is that $\mathbf{9}$ has no spiro junction for rings $\mathrm{D}$ and $\mathrm{E}$. The five-membered ether ring $\mathrm{D}$ and its connections with rings $\mathrm{C}$ and $\mathrm{E}$ were confirmed by ${ }^{1} \mathrm{H}-{ }^{1} \mathrm{H}$ COSY correlation (Table 1 and Figure 2$)$ of $\mathrm{H}-8\left(\delta_{\mathrm{H}} 3.12, \mathrm{~m}\right)$ with $\mathrm{H}-13\left(\delta_{\mathrm{H}}\right.$ $3.78, \mathrm{~d}, J=5.7 \mathrm{~Hz})$ and HMBC correlations (Table 1 and Figure 2$)$ of $\mathrm{H}-13$ with $\mathrm{C}-8\left(\delta_{\mathrm{C}} 48.9\right), \mathrm{C}-9\left(\delta_{\mathrm{C}}\right.$ 87.5), C-11 $\left(\delta_{\mathrm{C}} 126.8\right), \mathrm{C}-12\left(\delta_{\mathrm{C}} 48.6\right), \mathrm{C}-14\left(\delta_{\mathrm{C}} 102.1\right), \mathrm{C}-16\left(\delta_{\mathrm{C}} 139.2\right)$ and $\mathrm{C}-31\left(\delta_{\mathrm{C}} 22.0\right), \mathrm{H}-15\left(\delta_{\mathrm{H}} 4.35\right.$, q, $J=6.4 \mathrm{~Hz})$ with $\mathrm{C}-11$ and $\mathrm{C}-31$ and $\mathrm{H}-19\left(\delta_{\mathrm{H}} 6.95, \mathrm{~d}, J=1.8 \mathrm{~Hz}\right)$ with $\mathrm{C}-14, \mathrm{C}-17\left(\delta_{\mathrm{C}} 172.7\right)$ and $\mathrm{C}-18$ $\left(\delta_{\mathrm{C}}\right.$ 88.1). The locations of $\mathrm{CH}_{3}-31, \mathrm{CH}_{3}-32$ and $\mathrm{OH}-14$ were also established by HMBC correlations of $\mathrm{H}_{3}-31\left(\delta_{\mathrm{H}} 1.35, \mathrm{~s}\right)$ with C-11, C-12, C-13 $\left(\delta_{\mathrm{C}} 56.3\right)$ and C-15 $\left(\delta_{\mathrm{C}} 79.4\right)$ and $\mathrm{H}_{3}-32\left(\delta_{\mathrm{H}} 1.26, \mathrm{~d}, J=6.4 \mathrm{~Hz}\right)$ with C-12 and C-15, as well as $\mathrm{OH}-14\left(\delta_{\mathrm{H}} 6.25\right.$, s) with C-14 and C-16. The planner structure of 9 was further confirmed by $X$-ray diffraction analysis $(\mathrm{Cu} \mathrm{K} \alpha, \mathrm{CCDC}$ deposition number 1868814, crystallized in $\mathrm{MeOH}$, Figure 3).

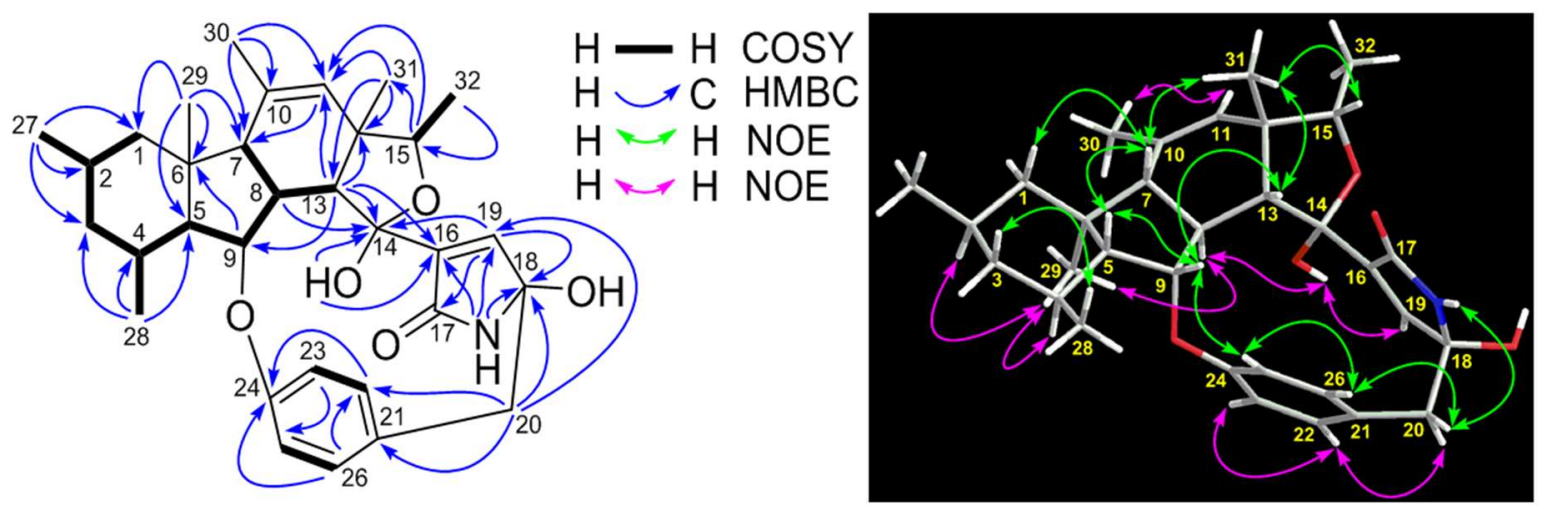

Figure 2. ${ }^{1} \mathrm{H}-{ }^{1} \mathrm{H}$ COSY, key HMBC and NOE correlations of penicipyrroether A (9).

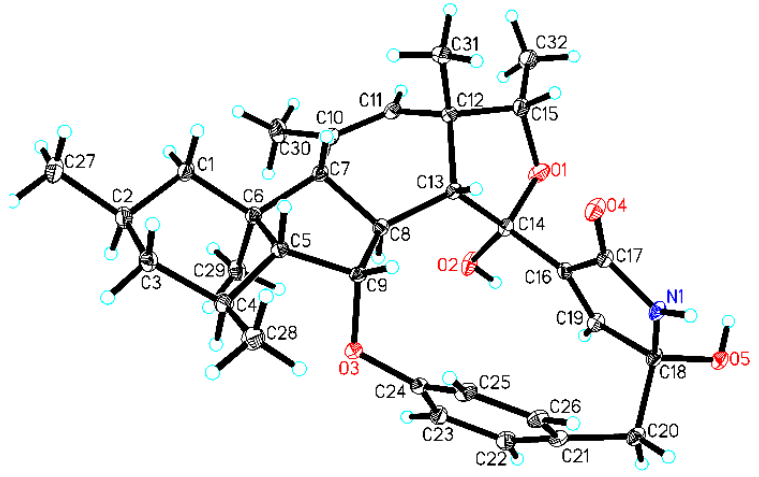

Figure 3. X-ray crystal structure of penicipyrroether $\mathrm{A}(9, \mathrm{Cu} \mathrm{K} \alpha$ radiation).

The relative stereochemistry of 9 was established based on the analyses of NOESY spectrum (Figures S18 and S19) and coupling constants. As depicted in Figure 2, NOE correlations of H-9 with $\mathrm{H}-5$ and $\mathrm{H}-13, \mathrm{H}_{3}-31$ with $\mathrm{H}-7, \mathrm{H}-13$ and $\mathrm{H}-15, \beta \mathrm{H}-20$ with $\mathrm{NH}-17$ and $\mathrm{H}-26$ and $\mathrm{H}-25$ with $\mathrm{H}-9$ and $\mathrm{H}-26$ indicated a $\beta$-orientation for these protons. Similarly, NOE correlations of $\mathrm{H}_{3}-29$ with $\mathrm{H}-2, \mathrm{H}-4$ and $\mathrm{H}-8, \mathrm{H}-8$ with $\mathrm{OH}-14$ and $\mathrm{H}-22$ with $\alpha \mathrm{H}-20$ and $\mathrm{H}-23$ were suggestive of an $\alpha$-orientation for these protons. The large coupling constants of ${ }^{3} J_{4,5}(11.3 \mathrm{~Hz})$ and ${ }^{3} J_{7,8}(13.2 \mathrm{~Hz})$ also confirmed the trans-juncture for $\mathrm{A} / \mathrm{B}$ and $\mathrm{B} / \mathrm{C}$ rings [16]. NOE correlations of $\mathrm{H}-11$ with $\mathrm{H}_{3}-30$ and $\mathrm{H}-19$ with $\mathrm{OH}-14$ 
indicated a Z-geometry for both of the double bonds at $C_{10-11}$ and $C_{16-19}$. The absolute configuration of 9 was determined as $2 R, 4 S, 5 R, 6 S, 7 S, 8 S, 9 S, 12 S, 13 R, 14 R, 18 R$ based on the result of the X-ray diffraction analysis $(\mathrm{Cu} \mathrm{K} \alpha$, Flack parameter 0.02). A computational method was also applied to assign the absolute stereochemistry of 9 by comparing its experimental electronic circular dichroism (ECD) spectrum with the calculated ECD spectra. The result (Figure 4) showed that the calculated ECD spectrum of model molecule 9a was close to the experimental curve of 9. Based on the foregoing evidence, the structure of 9 was elucidated as a new alkaloid, named penicipyrroether A. Its ${ }^{13} \mathrm{C}$ and ${ }^{1} \mathrm{H}$ NMR data (Table 1 ) were assigned based on a combination of HSQC, ${ }^{1} \mathrm{H}-{ }^{1} \mathrm{H}$ COSY, HMBC and NOE spectroscopic analyses.

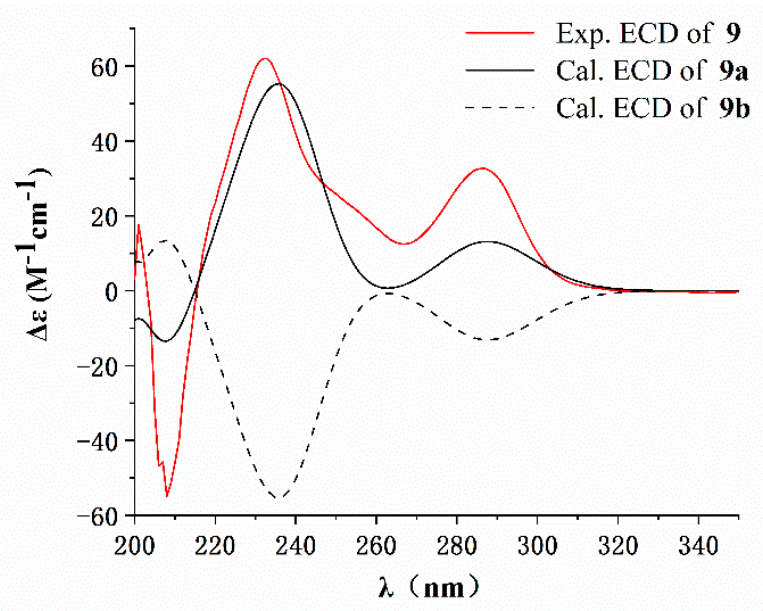

Figure 4. Experimental electronic circular dichroism (ECD) spectrum of penicipyrroether A (9, 200-350 nm) in $\mathrm{MeOH}$ and the calculated ECD spectra of the model molecules $\mathbf{9 a}$ and $\mathbf{9 b}$ at the B3LYP/6-311+G(d,p) level (9a: 2R,4S,5R,6S,7S,8S,9S,12S,13R,14R,18R; 9b: 2S,4R,5S,6R,7R,8R,9R,12R, $13 S, 14 S, 18 S)$.

It has been reported that compound 20, a dehydro-derivative of GKK1032A (19) (Figure 5), might be a precursor of the fungal metabolites of GKK1032s, pyrrospirones and penicipyrrodiether A [1-3,17]. Similarly, penicipyrroether A (9) might be also derived from 20. A proposed biosynthetic pathway for penicipyrroether A (9) was depicted in Figure 5. After oxidation of the vinyl group of 20, the epoxide derivative (21) was transformed into 22 and then an intramolecular hemiketal formation [3,15] of 22 forms penicipyrroether A (9).

Table $1 .{ }^{13} \mathrm{C}$ and ${ }^{1} \mathrm{H}$ NMR data of penicipyrroether $\mathrm{A}\left(\mathbf{9}\right.$, in pyridine- $\left.d_{5}\right)$.

\begin{tabular}{ccccc}
\hline No. & $\delta_{\mathbf{C}}$, Type & $\delta_{\mathbf{H}}(\boldsymbol{J}$ in Hz) & ${ }^{\mathbf{1}} \mathbf{H}^{-1} \mathbf{H}$ COSY & HMBC \\
\hline 1 & $49.2, \mathrm{CH}_{2}$ & $\begin{array}{c}\beta \mathrm{H}: 0.76, \mathrm{t}(12.0) ; \\
\alpha \mathrm{H}: 1.88, \mathrm{dd}(12.2,3.3)\end{array}$ & $\begin{array}{c}\mathrm{H}-2, \alpha \mathrm{H}-1 ; \\
\mathrm{H}-2, \beta \mathrm{H}-1\end{array}$ & $\mathrm{C}-27, \mathrm{C}-29$ \\
\hline 2 & $28.5, \mathrm{CH}$ & $1.76, \mathrm{~m}$ & $\mathrm{H}-1, \mathrm{H}-3, \mathrm{H}_{3}-27$ & \\
\hline 3 & $46.1, \mathrm{CH}_{2}$ & $\begin{array}{c}\beta \mathrm{H}: 0.57, \mathrm{q}(12.0) ; \\
\alpha \mathrm{H}: 1.69, \mathrm{~m}\end{array}$ & $\begin{array}{c}\mathrm{H}-2, \alpha \mathrm{H}-3, \mathrm{H}-4 ; \\
\mathrm{H}-2, \beta \mathrm{H}-3, \mathrm{H}-4\end{array}$ & $\mathrm{C}-27, \mathrm{C}-28$ \\
\hline 4 & $28.0, \mathrm{CH}$ & $1.97, \mathrm{~m}$ & $\mathrm{H}-3, \mathrm{H}-5, \mathrm{H}_{3}-28$ & \\
\hline 5 & $62.0, \mathrm{CH}$ & $1.22, \mathrm{dd}(11.3,7.6)$ & $\mathrm{H}-4, \mathrm{H}-9$ & $\mathrm{C}-4, \mathrm{C}-6, \mathrm{C}-29$ \\
\hline 6 & $41.3, \mathrm{C}$ & - & & \\
\hline 7 & $54.4, \mathrm{CH}$ & $2.36, \mathrm{~d}(13.2)$ & $\mathrm{H}-8$ & $\mathrm{C}-10$ \\
\hline 8 & $48.9, \mathrm{CH}$ & $3.12, \mathrm{~m}$ & $\mathrm{H}-7, \mathrm{H}-9, \mathrm{H}-13$ & $\mathrm{C}-7, \mathrm{C}-9, \mathrm{C}-13, \mathrm{C}-14$ \\
\hline 9 & $87.5, \mathrm{CH}$ & $5.01, \mathrm{dd}(7.6,4.8)$ & $\mathrm{H}-5, \mathrm{H}-8$ & $\mathrm{C}-6$ \\
\hline
\end{tabular}


Table 1. Cont.

\begin{tabular}{|c|c|c|c|c|}
\hline No. & $\delta_{\mathrm{C}}$, Type & $\delta_{\mathrm{H}}(J$ in $\mathrm{Hz})$ & ${ }^{1} \mathrm{H}_{-}{ }^{1} \mathrm{H}$ COSY & НМВС \\
\hline 10 & $139.9, \mathrm{C}$ & - & & \\
\hline 11 & $126.8, \mathrm{CH}$ & $5.62, \mathrm{~s}$ & & $C-7, C-30$ \\
\hline 12 & 48.6, C & - & & \\
\hline 13 & $56.3, \mathrm{CH}$ & $3.78, \mathrm{~d}(5.7)$ & $\mathrm{H}-8$ & $\begin{array}{l}\text { C-9, C-11, C-12, } \\
\text { C-14, C-16, C-31 }\end{array}$ \\
\hline 14 & 102.1, C & - & & \\
\hline 15 & $79.4, \mathrm{CH}$ & $4.35, \mathrm{q}(6.4)$ & $\mathrm{H}_{3}-32$ & $C-11, C-31$ \\
\hline 16 & $139.2, \mathrm{C}$ & - & & \\
\hline 17 & 172.7, C & - & & \\
\hline 18 & $88.1, \mathrm{C}$ & - & & \\
\hline 19 & 147.3, CH & $6.95, \mathrm{~d}(1.8)$ & & C-14, C-17, C-18 \\
\hline 20 & $45.7, \mathrm{CH}_{2}$ & $\begin{array}{l}\beta H: 3.56, d(12.2) ; \\
\alpha H: 3.51, d(12.2)\end{array}$ & $\begin{array}{l}\alpha \mathrm{H}-20 \\
\beta \mathrm{H}-20\end{array}$ & $\begin{array}{c}\text { C-18, C-19, C-21, } \\
\text { C-22, C-26 }\end{array}$ \\
\hline 21 & $130.8, \mathrm{C}$ & - & & \\
\hline 22 & 132.7, $\mathrm{CH}$ & 7.31, dd $(8.1,1.9)$ & $\mathrm{H}-23$ & C-20, C-24, C-26 \\
\hline 23 & $122.4, \mathrm{CH}$ & $7.13, \mathrm{dd}(8.1,2.4)$ & H-22 & $C-21, C-25$ \\
\hline 24 & 159.6, C & - & & \\
\hline 25 & $118.4, \mathrm{CH}$ & $7.22^{a}$ & H-26 & $C-21, C-23$ \\
\hline 26 & $130.2, \mathrm{CH}$ & 7.38, dd $(8.4,1.9)$ & H-25 & $\mathrm{C}-22, \mathrm{C}-24$ \\
\hline 27 & $23.4, \mathrm{CH}_{3}$ & $0.91, \mathrm{~d}(6.4)$ & $\mathrm{H}-2$ & $C-1, C-2, C-3$ \\
\hline 28 & $20.2, \mathrm{CH}_{3}$ & $1.18, \mathrm{~d}(6.3)$ & $\mathrm{H}-4$ & $C-3, C-4, C-5$ \\
\hline 29 & $16.8, \mathrm{CH}_{3}$ & $1.31, \mathrm{~s}$ & & $C-1, C-5, C-6, C-7$ \\
\hline 30 & $20.8, \mathrm{CH}_{3}$ & $1.81, \mathrm{~s}$ & & C-7, C-10, C-11 \\
\hline 31 & $22.0, \mathrm{CH}_{3}$ & $1.35, \mathrm{~s}$ & & $\begin{array}{c}\mathrm{C}-11, \mathrm{C}-12, \mathrm{C}-13, \\
\mathrm{C}-15\end{array}$ \\
\hline 32 & $14.8, \mathrm{CH}_{3}$ & $1.26, \mathrm{~d}(6.4)$ & H-15 & $C-12, C-15$ \\
\hline $\mathrm{OH}-14$ & - & $6.25, \mathrm{~s}$ & & $C-14, C-16$ \\
\hline $\mathrm{OH}-18$ & - & $8.35, \mathrm{~s}$ & & \\
\hline NH-17 & - & $9.43, \mathrm{~s}$ & & C-16, C-18, C-19 \\
\hline
\end{tabular}

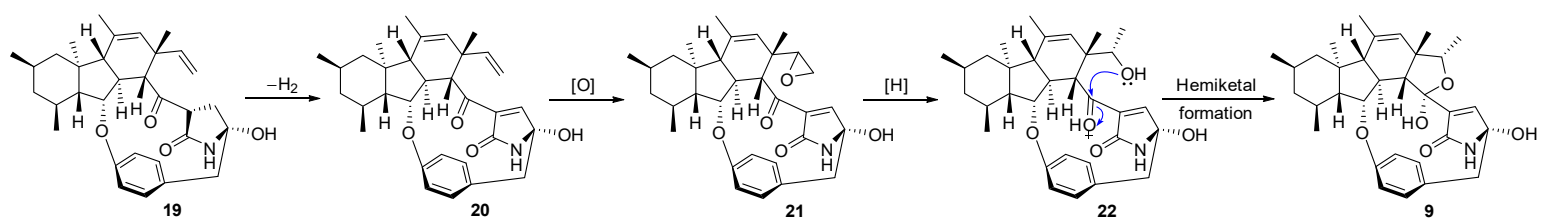

Figure 5. Plausible biosynthetic pathway of penicipyrroether A (9).

Compound 10 was obtained as a colorless amorphous powder and has a molecular formula $\mathrm{C}_{30} \mathrm{H}_{37} \mathrm{NO}_{6}$ deduced from its HRESIMS ions at $m / z[\mathrm{M}+\mathrm{H}]^{+} 508.2711$ and $[\mathrm{M}+\mathrm{Na}]^{+} 530.2531$ as well as its ${ }^{13} \mathrm{C}$ NMR data, which showed two fewer carbons, when compared to the structure of penicipyrroether A (9). Detailed interpretation of ${ }^{1} \mathrm{H},{ }^{13} \mathrm{C},{ }^{1} \mathrm{H}^{-1} \mathrm{H}$ COSY, HSQC and HMBC spectra (Figures S23-S38) demonstrated that compound $\mathbf{1 0}$ is an analogue of penicipyrroether A (9) with the 
same structural part of rings A, B, F and G but possesses an epoxy moiety at C-10 and C-11 and a different five-membered ether ring $\mathrm{D}$ fused with ring $\mathrm{E}$ through a spiro carbon of $\mathrm{C}-15$. In addition, the dehydro-pyrrolidinone moiety (ring E) in $\mathbf{9}$ was replaced by a pyrrolidinone moiety in $\mathbf{1 0}$. The presence of the epoxy moiety was confirmed by HMBC correlations (Table 2 and Figure 6) of H-7 $\left(\delta_{\mathrm{H}} 1.55, \mathrm{~d}, J=14.3 \mathrm{~Hz}\right)$ with $\mathrm{C}-10\left(\delta_{\mathrm{C}} 58.8\right)$ and C-11 $\left(\delta_{\mathrm{C}} 63.5\right), \mathrm{H}-11\left(\delta_{\mathrm{H}} 2.46, \mathrm{~s}\right)$ with C-10, $\mathrm{C}-12\left(\delta_{\mathrm{C}}\right.$ 81.3), C-13 $\left(\delta_{C} 44.5\right), \mathrm{C}-29\left(\delta_{\mathrm{C}} 21.0\right)$ and $\mathrm{C}-30\left(\delta_{\mathrm{C}} 26.0\right)$ and $\mathrm{H}_{3}-29\left(\delta_{\mathrm{H}} 1.20, \mathrm{~s}\right)$ with C-7 $\left(\delta_{\mathrm{C}} 49.9\right), \mathrm{C}-10$ and $\mathrm{C}-11$. Similarly, the ether ring $\mathrm{D}$ and its connections with rings $\mathrm{C}$ and $\mathrm{E}$ was indicated by HMBC correlations of $\mathrm{H}-8\left(\delta_{\mathrm{H}} 2.79, \mathrm{~m}\right)$ with $\mathrm{C}-13$ and $\mathrm{C}-14\left(\delta_{\mathrm{C}} 180.9\right), \mathrm{H}-11$ with $\mathrm{C}-12$ and $\mathrm{C}-13, \mathrm{H}-13\left(\delta_{\mathrm{H}} 3.21\right.$, $\mathrm{d}, J=8.0 \mathrm{~Hz})$ with C-8 $\left(\delta_{\mathrm{C}} 39.2\right), \mathrm{C}-11, \mathrm{C}-12, \mathrm{C}-14$ and C-30, H-18 $\left(\delta_{\mathrm{H}} 1.99,2.45, \mathrm{~d}, J=12.1 \mathrm{~Hz}\right.$, each $)$ with C-14, C-15 $\left(\delta_{\mathrm{C}} 79.7\right), \mathrm{C}-16\left(\delta_{\mathrm{C}} 172.1\right), \mathrm{C}-17\left(\delta_{\mathrm{C}} 86.2\right)$ and C-19 $\left(\delta_{\mathrm{C}} 45.0\right), \mathrm{H}_{3}-30\left(\delta_{\mathrm{H}} 1.59, \mathrm{~s}\right)$ with C-11, C-12 and C-13 and NH-16 $\left(\delta_{\mathrm{H}} 8.76\right.$, s) with C-15, C-16, C-17 and C-18 $\left(\delta_{\mathrm{C}} 40.9\right)$.

Table 2. ${ }^{13} \mathrm{C}$ and ${ }^{1} \mathrm{H}$ NMR data of pyrrospirone J (10, in DMSO- $\left.d_{6}\right)$.

\begin{tabular}{|c|c|c|c|c|}
\hline No. & $\delta_{\mathrm{C}}$, Type & $\delta_{\mathrm{H}}(J$ in $\mathrm{Hz})$ & ${ }^{1} \mathrm{H}_{-1}{ }^{1} \mathrm{H}$ COSY & HМBC \\
\hline 1 & 47.3, $\mathrm{CH}_{2}$ & $\begin{array}{c}\beta \mathrm{H}: 0.81, \mathrm{t}(12.3) \\
\alpha \mathrm{H}: 1.80, \mathrm{~m}\end{array}$ & $\begin{array}{l}\mathrm{H}-2, \alpha \mathrm{H}-2 ; \\
\mathrm{H}-2, \beta \mathrm{H}-2\end{array}$ & $C-26, C-28$ \\
\hline 2 & $27.4, \mathrm{CH}$ & $1.82, \mathrm{~m}$ & $\mathrm{H}-1, \mathrm{H}-3, \mathrm{H}_{3}-26$ & C-6 \\
\hline 3 & $45.5, \mathrm{CH}_{2}$ & $\begin{array}{c}\beta \mathrm{H}: 0.51, \mathrm{q}(12.1) \\
\alpha \mathrm{H}: 1.75, \mathrm{~m}\end{array}$ & $\begin{array}{l}\mathrm{H}-2, \alpha \mathrm{H}, \mathrm{H}-4 \\
\mathrm{H}-2, \beta \mathrm{H}, \mathrm{H}-4\end{array}$ & $\mathrm{C}-2, \mathrm{C}-27$ \\
\hline 4 & $26.8, \mathrm{CH}$ & $1.78, \mathrm{~m}$ & $\mathrm{H}-3, \mathrm{H}-5, \mathrm{H}_{3}-27$ & $\mathrm{C}-2$ \\
\hline 5 & $59.5, \mathrm{CH}$ & $1.22, \mathrm{dd}(11.6,8.8)$ & $\mathrm{H}-4, \mathrm{H}-9$ & C-4, C-6, C-28 \\
\hline 6 & $42.5, \mathrm{C}$ & - & & \\
\hline 7 & $49.9, \mathrm{CH}$ & $1.55, \mathrm{~d}(14.3)$ & $\mathrm{H}-8$ & $\begin{array}{c}\text { C-1, C-5, C-6, C-8, } \\
\text { C-13, C-28 }\end{array}$ \\
\hline 8 & $39.2, \mathrm{CH}$ & $2.79, \mathrm{~m}$ & H-7, H-9, H-13 & $\begin{array}{c}\text { C-7, C-9, C-10, } \\
\text { C-13, C-14 }\end{array}$ \\
\hline 9 & $85.0, \mathrm{CH}$ & $4.82, \mathrm{dd}(8.6,7.0)$ & H-5, H-8 & C-5, C-6, C-13, C-23 \\
\hline 10 & $58.8, \mathrm{C}$ & - & & \\
\hline 11 & $63.5, \mathrm{CH}$ & $2.46, \mathrm{~s}$ & & $\begin{array}{c}\mathrm{C}-10, \mathrm{C}-12, \mathrm{C}-13, \\
\mathrm{C}-29, \mathrm{C}-30\end{array}$ \\
\hline 12 & $81.3, \mathrm{C}$ & - & & \\
\hline 13 & $44.5, \mathrm{CH}$ & $3.21, \mathrm{~d}(8.0)$ & & $\begin{array}{c}\text { C-8, C-9, C-11, } \\
\text { C-12, C-14, C-30 }\end{array}$ \\
\hline 14 & $180.9, \mathrm{C}$ & - & & \\
\hline 15 & 79.7, C & - & & \\
\hline 16 & 172.1, C & - & & \\
\hline 17 & $86.2, \mathrm{C}$ & - & & \\
\hline 18 & $40.9, \mathrm{CH}_{2}$ & $\begin{array}{l}\beta \mathrm{H}: 1.99, \mathrm{~d}(12.1) \\
\alpha \mathrm{H}: 2.45, \mathrm{~d}(12.1)\end{array}$ & $\begin{array}{l}\alpha \mathrm{H}-18 ; \\
\beta \mathrm{H}-18\end{array}$ & $\begin{array}{c}\text { C-14, C-15, C-16, } \\
\text { C-17, C-19 }\end{array}$ \\
\hline 19 & $45.0, \mathrm{CH}_{2}$ & $\begin{array}{l}\beta \mathrm{H}: 2.99, \mathrm{~d}(14.5) \\
\alpha \mathrm{H}: 2.69, \mathrm{~d}(14.5)\end{array}$ & $\begin{array}{l}\alpha \mathrm{H}-19 ; \\
\beta \mathrm{H}-19\end{array}$ & $\begin{array}{c}\text { C-17, C-18, C-20, } \\
\text { C- } 21, \text { C- } 25\end{array}$ \\
\hline 20 & $129.4, \mathrm{C}$ & - & & \\
\hline 21 & $133.3, \mathrm{CH}$ & $6.92, \mathrm{dd}(8.3,2.1)$ & $\mathrm{H}-22$ & C-19, C-23, C-25 \\
\hline 22 & 124.4, $\mathrm{CH}$ & $6.82, \mathrm{dd}(8.3,2.7)$ & $\mathrm{H}-21$ & C-20, C-23, C-24 \\
\hline 23 & $158.7, \mathrm{C}$ & - & & \\
\hline
\end{tabular}


Table 2. Cont.

\begin{tabular}{ccccc}
\hline No. & $\boldsymbol{\delta}_{\mathrm{C}}$, Type & $\boldsymbol{\delta}_{\mathbf{H}}(\boldsymbol{J}$ in $\mathbf{H z})$ & ${ }^{\mathbf{1}} \mathbf{H}-{ }^{\mathbf{1}} \mathbf{H}$ COSY & HMBC \\
\hline 24 & $119.7, \mathrm{CH}$ & $6.94, \mathrm{dd}(8.8,2.7)$ & $\mathrm{H}-25$ & $\mathrm{C}-20, \mathrm{C}-22, \mathrm{C}-23$ \\
\hline 25 & $130.6, \mathrm{CH}$ & $6.78, \mathrm{dd}(8.8,2.1)$ & $\mathrm{H}-24$ & $\mathrm{C}-19, \mathrm{C}-21, \mathrm{C}-23$ \\
\hline 26 & $22.7, \mathrm{CH}_{3}$ & $0.86, \mathrm{~d}(6.1)$ & $\mathrm{H}-2$ & $\mathrm{C}-1, \mathrm{C}-2, \mathrm{C}-3$ \\
\hline 27 & $19.7, \mathrm{CH}_{3}$ & $1.02, \mathrm{~d}(6.2)$ & $\mathrm{H}-4$ & $\mathrm{C}-3, \mathrm{C}-4, \mathrm{C}-5$ \\
\hline 28 & $15.2, \mathrm{CH}_{3}$ & $1.05, \mathrm{~s}$ & & $\mathrm{C}-1, \mathrm{C}-5, \mathrm{C}-6, \mathrm{C}-7$ \\
\hline 29 & $21.0, \mathrm{CH}_{3}$ & $1.20, \mathrm{~s}$ & $\mathrm{C}-7, \mathrm{C}-10, \mathrm{C}-11$ \\
\hline 30 & $26.0, \mathrm{CH}_{3}$ & $1.59, \mathrm{~s}$ & $\mathrm{C}-11, \mathrm{C}-12, \mathrm{C}-13$ \\
\hline $\mathrm{OH}-17$ & - & $6.24, \mathrm{~s}$ & $\mathrm{C}-17, \mathrm{C}-18, \mathrm{C}-19$ \\
\hline $\mathrm{NH}-16$ & - & $8.76, \mathrm{~s}$ & $\mathrm{C}-15, \mathrm{C}-16, \mathrm{C}-17$, \\
\hline
\end{tabular}
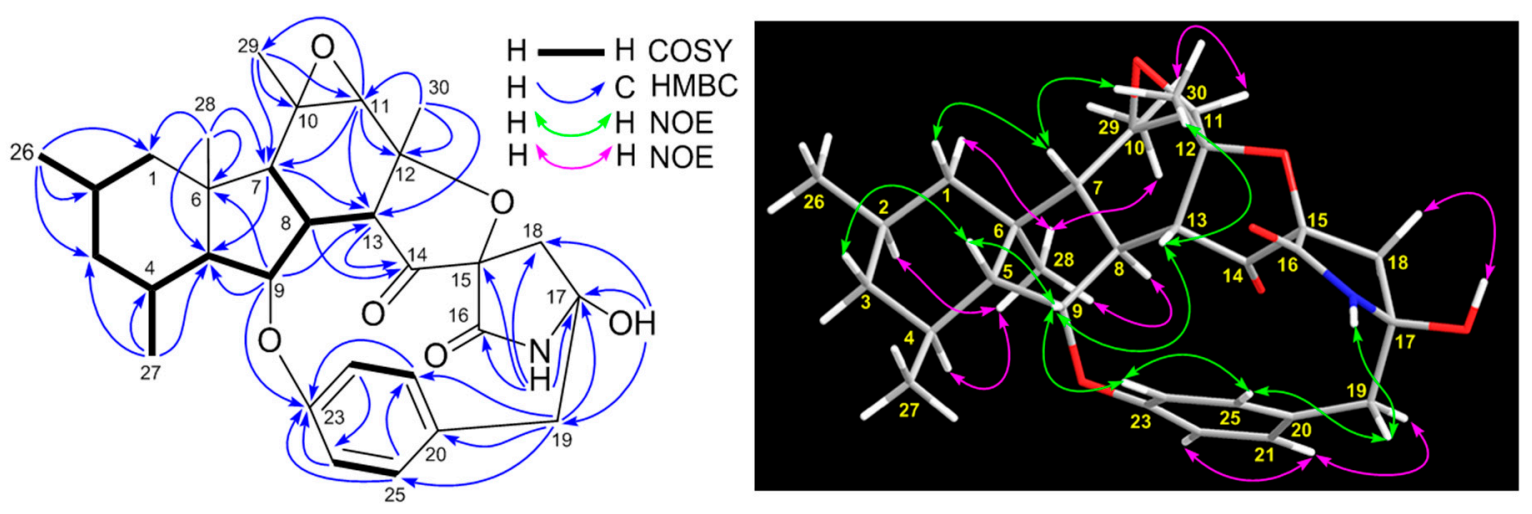

Figure 6. ${ }^{1} \mathrm{H}-{ }^{1} \mathrm{H}$ COSY, key HMBC and NOE correlations of pyrrospirone $\mathrm{J}(\mathbf{1 0})$.

The relative stereochemistry of $\mathbf{1 0}$ was assigned by analyses of the NOESY spectrum (Figures S39 and S40) and coupling constants. NOE correlations (Figure 6$)$ of $\mathrm{H}-5\left(\delta_{\mathrm{H}} 1.22\right)$ with $\beta \mathrm{H}-3\left(\delta_{\mathrm{H}} 0.51\right)$ and $\mathrm{H}-9\left(\delta_{\mathrm{H}} 4.82\right), \mathrm{H}-7\left(\delta_{\mathrm{H}} 1.55\right)$ with $\beta \mathrm{H}-1\left(\delta_{\mathrm{H}} 0.81\right)$ and $\mathrm{H}_{3}-30\left(\delta_{\mathrm{H}} 1.59\right), \mathrm{H}-9$ with $\mathrm{H}-13\left(\delta_{\mathrm{H}} 3.21\right)$ and $\mathrm{H}-24\left(\delta_{\mathrm{H}} 6.94\right), \mathrm{H}-13$ with $\mathrm{H}_{3}-30, \mathrm{NH}-16\left(\delta_{\mathrm{H}} 8.76\right)$ with $\beta \mathrm{H}-19\left(\delta_{\mathrm{H}} 2.99\right)$ and $\mathrm{H}-25\left(\delta_{\mathrm{H}} 6.78\right)$ and $\mathrm{H}-25$ with $\beta \mathrm{H}-19$ and $\mathrm{H}-24$ suggested a $\beta$-orientation for these protons; while NOE correlations of $\mathrm{H}-8\left(\delta_{\mathrm{H}}\right.$ 2.79) with $\mathrm{H}_{3}-28\left(\delta_{\mathrm{H}} 1.05\right), \mathrm{H}_{3}-28$ with $\alpha \mathrm{H}-1\left(\delta_{\mathrm{H}} 1.80\right), \mathrm{H}-2\left(\delta_{\mathrm{H}} 1.82\right), \mathrm{H}-4\left(\delta_{\mathrm{H}} 1.78\right)$ and $\mathrm{H}_{3}-29\left(\delta_{\mathrm{H}} 1.20\right)$, $\mathrm{H}_{3}-29$ with $\mathrm{H}-11\left(\delta_{\mathrm{H}} 2.46\right), \mathrm{H}-21\left(\delta_{\mathrm{H}} 6.92\right)$ with $\alpha \mathrm{H}-19\left(\delta_{\mathrm{H}} 2.69\right)$ and $\mathrm{H}-22\left(\delta_{\mathrm{H}} 6.82\right)$ and $\mathrm{H}-18\left(\delta_{\mathrm{H}} 1.99\right)$ with $\mathrm{OH}-17\left(\delta_{\mathrm{H}} 6.24\right)$ proved an $\alpha$-orientation for these protons. The trans-juncture for $\mathrm{A} / \mathrm{B}$ and $\mathrm{B} / \mathrm{C}$ rings was also confirmed by the large coupling constants of ${ }^{3} J_{4,5}(11.6 \mathrm{~Hz})$ and ${ }^{3} J_{7,8}(14.3 \mathrm{~Hz})$ [16]. The absolute configuration of 10 was determined as $2 R, 4 S, 5 R, 6 R, 7 R, 8 S, 9 S, 10 R, 11 R, 12 R, 13 R, 15 R, 17 R$ based on the result from ECD calculation (Figure 7). Unfortunately, several efforts to obtain single crystal for X-ray diffraction were not successful because the structure of $\mathbf{1 0}$ was changed during the process of crystallization.

It was noted that the ketonic carbonyl at C-14 of $\mathbf{1 0}$ resonated at $180.9 \mathrm{ppm}$, which is unusual. In order to confirm this possibility, we conducted a computational calculation of its ${ }^{13} \mathrm{C}$ chemical shifts [18-20]. The calculated ${ }^{13} \mathrm{C}$ NMR data (Table S7) of 10 were in good agreement with its experimental values, with the corrected mean absolute error (CMAE) of $0.28 \mathrm{ppm}$ and the correlation coefficient $\left(R^{2}\right)$ of 0.9982 (Figure 8 ) and the calculated ${ }^{13} \mathrm{C}$ chemical shift for $\mathrm{C}-14$ was $184.1 \mathrm{ppm}$, which was close to the experimental value of $180.9 \mathrm{ppm}$. Based on the foregoing evidence, the structure of $\mathbf{1 0}$ was determined as a new alkaloid, named pyrrospirone J. The full assignment of ${ }^{13} \mathrm{C}$ and ${ }^{1} \mathrm{H}$ NMR data (Table 2) was made based on HSQC, ${ }^{1} \mathrm{H}^{-1} \mathrm{H}$ COSY, HMBC and NOE spectroscopic analyses. 


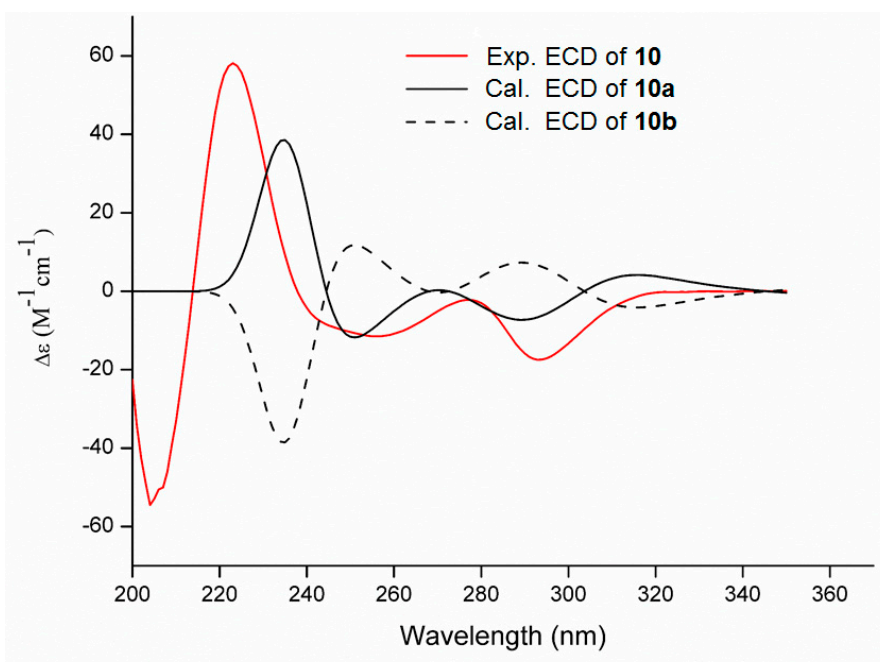

Figure 7. Experimental ECD spectrum of pyrrospirone $\mathrm{J}(\mathbf{1 0}, \mathbf{2 0 0}-400 \mathrm{~nm})$ in $\mathrm{MeOH}$ and the calculated ECD spectra of the model molecules 10a and 10b at the B3LYP/6-311+G(d,p) level (10a: $2 R, 4 S, 5 R, 6 R, 7 R, 8 S, 9 S, 10 R, 11 R, 12 R, 13 R, 15 R, 17 R ; 10 \mathbf{b}: 2 S, 4 R, 5 S, 6 S, 7 S, 8 R, 9 R, 10 S, 11 S, 12 S, 13 S, 15 S, 17 S)$.

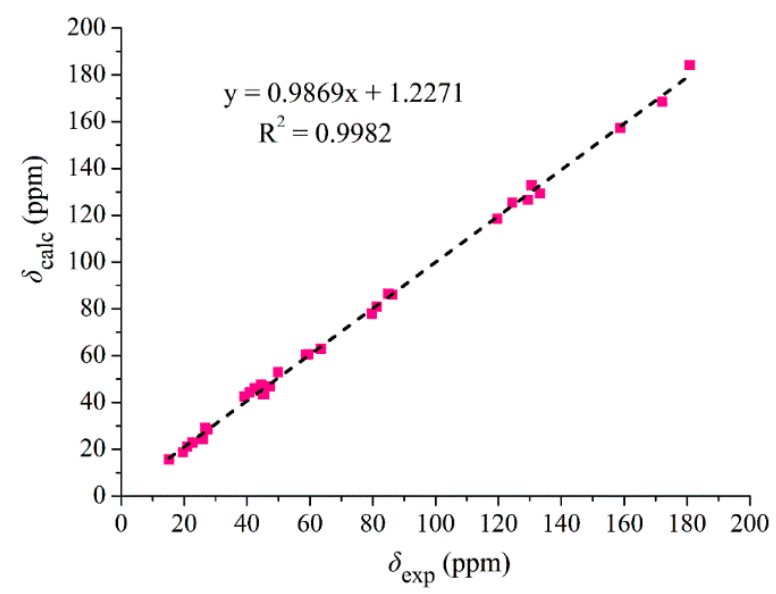

Figure 8. Regression analysis of experimental versus calculated ${ }^{13} \mathrm{CNMR}$ chemical shifts of pyrrospirone $\mathrm{J}(\mathbf{1 0})$ at the mPW1PW91-SCRF (DMSO)/6-311+g(d,p) level and linear fitting is shown as a line.

New alkaloids-penicipyrroether A (9) and pyrrospirone J (10)—were evaluated for their activities in inhibiting the proliferation of human glioma U87MG and U251 cells through sulforhodamine B (SRB) assay [21]. Doxorubicin (DOX, a chemotherapeutic drug) was used as a positive control. It has been found that both compounds had activity against different glioma cells with $\mathrm{IC}_{50}$ values of 1.64-5.50 $\mu \mathrm{M}$ for 9 and 10.52-17.92 $\mu \mathrm{M}$ for $\mathbf{1 0}$ (Table 3). The cytotoxicities of penicipyrroether A (9) and DOX against normal human astrocytes were also tested and showed $\mathrm{CC}_{50}$ values of $23.28 \pm 1.05 \mu \mathrm{M}$ for 9 and $8.57 \pm 0.16 \mu \mathrm{M}$ for DOX. The data indicated that the antiglioma activity of 9 was equivalent to (or slightly stronger than) the activity of the positive control DOX and the selective index $\left(\mathrm{CC}_{50} / \mathrm{IC}_{50}\right)$ of 4.2-14.2 for 9 was higher than that of 1.1-7.1 for DOX.

The antibacterial activities of penicipyrroether A (9) and pyrrospirone J (10) against MRSA and E. coli were also determined by the micro broth dilution method [22]. Gentamicin (an antibiotic against both Gram-positive and Gram-negative bacteria) and vancomycin (an antibiotic against MRSA) were used as positive controls. The results (Table 3) showed that penicipyrroether A (9) had good antibacterial activities with MIC values of $1.7 \mu \mathrm{g} / \mathrm{mL}$ against MRSA and $3.0 \mu \mathrm{g} / \mathrm{mL}$ against E. coli. However, pyrrospirone J (10) was inactive at a concentration of $50 \mu \mathrm{g} / \mathrm{mL}$. 
Table 3. Antiglioma and antibacterial activities of penicipyrroether A (9) and pyrrospirone J (10).

\begin{tabular}{ccccc}
\hline \multirow{2}{*}{ Compounds } & \multicolumn{2}{c}{ Glioma Cells $(\mu \mathrm{M})$} & \multicolumn{2}{c}{ Bacteria $(\mu \mathrm{g} / \mathrm{mL})$} \\
\cline { 2 - 5 } & U87MG & U251 & MRSA & E. coli \\
\hline $\mathbf{9}$ & $1.64 \pm 0.05$ & $5.50 \pm 0.12$ & 1.7 & 3.0 \\
$\mathbf{1 0}$ & $10.52 \pm 0.62$ & $17.92 \pm 0.93$ & $>50$ & $>50$ \\
DOX & $1.20 \pm 0.06$ & $8.03 \pm 1.20$ & $\mathrm{NT}$ & $\mathrm{NT}$ \\
Gentamicin & $\mathrm{NT}$ & $\mathrm{NT}$ & 0.36 & 1.44 \\
Vancomycin & $\mathrm{NT}$ & $\mathrm{NT}$ & 0.20 & $\mathrm{NT}$ \\
\hline
\end{tabular}

NT: No testing.

\section{Materials and Methods}

\subsection{General Experimental Procedures}

Optical rotation, ultraviolet-visible (UV) and electronic circular dichroism (ECD) were measured on an Autopol I polarimeter (Rudolph Research Analytical, Hackettstown, NJ, USA), a METASH UV-8000 spectrometer (Shanghai METASH Instruments Co. Ltd., Shanghai, China) and a JASCO J-815 spectropolarimeter (JASCO Co. Tokyo, Japan), respectively. Infrared radiation (IR) spectra were recorded on a Bruker TENSOR II high performance FT-IR spectrometer (Bruker, Karlsruhe, Germany). HRESIMS data was obtained from an Agilent 6230 time-of-flight liquid chromatography-mass spectrometry (TOF LC-MS, Agilent, CA, USA). NMR spectra were acquired on a Bruker 500 spectrometer and chemical shifts were expressed in $\delta$ (ppm). Octadecyl silane (ODS, Cosmosil $75 \mathrm{C}_{18}$-Prep, Nacalai Tesque Inc., Kyoto, Japan) was applied for column chromatography. HPLC separation was conducted on a SHIMADZU LC-20AP prepared HPLC system with column A (Welch-20, $250 \times 21 \mathrm{~mm}, 5 \mu \mathrm{m}$, $\left.\mathrm{XB}-\mathrm{C}_{18}\right)$ or column B $\left(\mathrm{CT}-30,280 \times 30 \mathrm{~mm}, 10 \mu \mathrm{m}\right.$, Fuji-C $\left.\mathrm{C}_{18}\right)$. All solvents were ordered from the Sinopharm Chemical Reagent Co. Ltd. (Shanghai, China). Human glioma U87MG (JDS-2568) and U251 (XB-0439) cells were purchased from the Cell Bank of the Chinese Academy of Sciences and normal human astrocytes (HA, Cat. No. 1800) from the ScienCell. Methicillin-resistant Staphylococcus aureus (MRSA) ATCC 43300 and Escherichia coli ATCC 25922 were gifts from Drs. Zhongjun Ma and Pinmei Wang, respectively. Gentamicin $(99.6 \%)$ and vancomycin $(>98.0 \%)$ were bought from Meilune Biotechnology Co. Ltd. (Dalian, China), doxorubicin (DOX, >98.0\%) from Sigma-Aldrich, PDA (potato dextrose agar) from Baisi Biotechnology Co. Ltd. (Hangzhou, China) and MHB (Mueller-Hinton Broth) from Oxoid Ltd. PDB medium (potato dextrose broth, potato $100 \mathrm{~g}$, glucose $10 \mathrm{~g}$, sea salt $35 \mathrm{~g}$, tap water $1000 \mathrm{~mL}$ ) was made in the authors' laboratory.

\subsection{Marine Strain ZZ380}

Strain ZZ380 was previously isolated from marine crab Pachygrapsus crassipes and assigned as Penicillium sp. ZZ380 by ITS DNA sequence analysis [2].

\subsection{Mass Culture of Strain ZZ380}

Colonies of the strain ZZ380 from the PDA slant were inoculated into $250 \mathrm{~mL}$ of PDB liquid medium in a $500 \mathrm{~mL}$ Erlenmeyer flask and then incubated at $28^{\circ} \mathrm{C}$ for three days on a rotary shaker (180 rpm) to produce seed broth. The seed broth of $5 \mathrm{~mL}$ was inoculated into a $500 \mathrm{~mL}$ Erlenmeyer flask, containing $250 \mathrm{~mL}$ of PDB medium and then incubated at $28^{\circ} \mathrm{C}$ for 30 days under stationary state. A total of $55 \mathrm{~L}$ of culture were prepared for this study.

\subsection{Isolation of Compounds $9-18$}

The $55 \mathrm{~L}$ culture of ZZ380 in PDB medium was centrifuged to give mycelia and broth. The mycelia were extracted with $\mathrm{MeOH}$ three times to get a $\mathrm{MeOH}$ extract. The broth was partitioned with EtOAc three times to give an EtOAc extract. A mixture $(23.0 \mathrm{~g})$ of the $\mathrm{MeOH}$ and EtOAc extract was 
fractionated by an ODS column eluting successively with $40 \%, 60 \%, 80 \%$ and $100 \% \mathrm{MeOH}$ to afford four fractions (Frs. 1-4). Fr. 1 was further separated by an ODS column eluting with $35 \%, 45 \%$ and $55 \% \mathrm{MeOH}$ to give three sub fractions (SFrs. 1a-1c). Through purification using prepared HPLC with column A at a flow rate of $12 \mathrm{~mL} / \mathrm{min}$, compounds $\mathbf{1 1}\left(4.2 \mathrm{mg}\right.$, $\mathrm{t}_{\mathrm{R}}: 22.0 \mathrm{~min}$, mobile phase: $\mathrm{MeOH} / \mathrm{H}_{2} \mathrm{O}$, 38/62, detector: $254 \mathrm{~nm})$ and $12\left(6.7 \mathrm{mg}, \mathrm{t}_{\mathrm{R}}: 31.0 \mathrm{~min}\right.$, mobile phase: $\mathrm{MeOH} / \mathrm{H}_{2} \mathrm{O}, 38 / 62$, detector: 254 $\mathrm{nm})$ were obtained from SFr. 1a; compounds $15\left(4.0 \mathrm{mg}\right.$, $\mathrm{t}_{\mathrm{R}}: 33.0 \mathrm{~min}$, mobile phase: $\mathrm{MeOH} / \mathrm{H}_{2} \mathrm{O}$, 45/55, detector: $210 \mathrm{~nm}), \mathbf{1 4}\left(20.5 \mathrm{mg}, \mathrm{t}_{\mathrm{R}}: 37.0 \mathrm{~min}\right.$, mobile phase: $\mathrm{MeOH} / \mathrm{H}_{2} \mathrm{O}, 53 / 47$, detector: $254 \mathrm{~nm}$ ), 18 (7.0 mg, $\mathrm{t}_{\mathrm{R}}: 37.0 \mathrm{~min}$, mobile phase: $\mathrm{MeOH} / \mathrm{H}_{2} \mathrm{O}, 66 / 34$, detector: $254 \mathrm{~nm}$ ) were obtained from SFr. 1b, SFr. 1c and Fr. 2, respectively. Fr. 3 and Fr. 4 were also separated by ODS column eluting with $85 \%$ or $95 \% \mathrm{MeOH}$ to furnish SFrs. $3 \mathrm{a}$ and $3 \mathrm{~b}$ or SFrs. $4 \mathrm{a}$ and $4 \mathrm{~b}$. Through purification using prepared HPLC with column $B$ at a flow rate of $15 \mathrm{~mL} / \mathrm{min}$, compounds 13 (43.0 mg, $\mathrm{t}_{\mathrm{R}}: 19.0 \mathrm{~min}$, mobile phase: $\mathrm{MeOH} / \mathrm{H}_{2} \mathrm{O}, 80 / 20$, detector: $210 \mathrm{~nm}$ ) and $17\left(9.5 \mathrm{mg}\right.$, $\mathrm{t}_{\mathrm{R}}: 45.0 \mathrm{~min}$, mobile phase: $\mathrm{MeOH} / \mathrm{H}_{2} \mathrm{O}, 83 / 17$, detector: $254 \mathrm{~nm})$ were obtained from SFrs. $3 \mathrm{a}$ and $3 \mathrm{~b}$, respectively; while compounds $9\left(3.1 \mathrm{mg}\right.$, $\mathrm{t}_{\mathrm{R}}$ : $41.0 \mathrm{~min}$, mobile phase: $\mathrm{MeOH} / \mathrm{H}_{2} \mathrm{O}, 91 / 9$, detector: $\left.210 \mathrm{~nm}\right)$ and $16\left(50.0 \mathrm{mg}, \mathrm{t}_{\mathrm{R}}: 62.0 \mathrm{~min}\right.$, mobile phase: $\mathrm{MeOH} / \mathrm{H}_{2} \mathrm{O}, 98 / 2 ; 280 \mathrm{~nm} ; \mathrm{t}_{\mathrm{R}} 62 \mathrm{~min} ; 50.0 \mathrm{mg}$ ) were obtained from SFrs. 4a and $4 \mathrm{~b}$, respectively.

Compound 10 was isolated from a previous culture of strain ZZ380 in BMPM medium [2]. A crude extract was fractionated on an ODS column eluting successively with $80 \% \mathrm{MeOH}, 90 \% \mathrm{MeOH}$ and $100 \% \mathrm{MeOH}$ to give six fractions (Frs. 1-6) based on the results of TLC analysis. Fr. 2 was re-separated by an ODS column eluting with $80 \% \mathrm{MeOH}$ to give Frs. 2A and 2B. Fr. 2A was further separated by prepared HPLC with column $B$ using mobile phase of acetonitrile/water (68:32) at a flow rate of $10 \mathrm{~mL} / \mathrm{min}$ to give $\mathbf{1 0}\left(2.0 \mathrm{mg}, \mathrm{t}_{\mathrm{R}}: 60.0 \mathrm{~min}\right)$ and Frs. $2 \mathrm{~A}_{1}-2 \mathrm{~A}_{3}$.

Penicipyrroether A (9): Colorless block crystals; molecular formula $\mathrm{C}_{32} \mathrm{H}_{41} \mathrm{NO}_{5} ;[\alpha]_{\mathrm{D}}^{20}+71.4^{\circ}$ (c 0.10, MeOH); ECD $(10 \mathrm{mg} / \mathrm{L}, \mathrm{MeOH}) \lambda_{\max }(\Delta \varepsilon) 208$ (-57.22), $233(+64.65), 286(+34.08) \mathrm{nm} ; \mathrm{UV}$ $(\mathrm{MeOH}) \lambda_{\max }(\log \varepsilon) 202$ (4.20), 229 (3.66), 278 (2.67) nm; IR (MeOH) $v_{\max }$ 3375, 2924, 1672, 1437, 1408, $1239,1098,1013,951 \mathrm{~cm}^{-1} ;{ }^{13} \mathrm{C}(125 \mathrm{MHz})$ and ${ }^{1} \mathrm{H}(500 \mathrm{MHz}) \mathrm{NMR}$ data, see Table 1 ; HRESIMS $\mathrm{m} / \mathrm{z}$ $[\mathrm{M}+\mathrm{H}]^{+} 520.3063$ (calcd. for $\mathrm{C}_{32} \mathrm{H}_{42} \mathrm{NO}_{5}, 520.3063$ ), $[\mathrm{M}+\mathrm{Na}]^{+} 542.2877$ (calcd. for $\mathrm{C}_{32} \mathrm{H}_{41} \mathrm{NNaO}_{5}$, 542.2882).

Pyrrospirone J (10): Colorless amorphous powder; molecular formula $\mathrm{C}_{30} \mathrm{H}_{37} \mathrm{NO}_{6}$; $\mathrm{ECD}(10 \mathrm{mg} / \mathrm{L}$, $\mathrm{MeOH}) \lambda_{\max }(\Delta \varepsilon) 204$ (-55.93), $223(+59.59), 256(-11.80), 293(-17.92) \mathrm{nm} ; \mathrm{UV}(\mathrm{MeOH}) \lambda_{\max }(\log \varepsilon)$ 211 (4.40), 230 (4.21), 284 (3.36) nm; IR (MeOH) $v_{\max }$ 3347, 2923, 2851, 1748, 1686, 1505, 1460, 1371, 1239, $1169,1074,937 \mathrm{~cm}^{-1} ;{ }^{13} \mathrm{C}(125 \mathrm{MHz})$ and ${ }^{1} \mathrm{H}(500 \mathrm{MHz}) \mathrm{NMR}$ data, see Table 2; HRESIMS $\mathrm{m} / z$ [M + H] ${ }^{+}$ 508.2711 (calcd. for $\mathrm{C}_{30} \mathrm{H}_{38} \mathrm{NO}_{6} 508.2699$ ) and $[\mathrm{M}+\mathrm{Na}]^{+} 530.2531$ (calcd. for $\mathrm{C}_{30} \mathrm{H}_{37} \mathrm{NNaO}_{6} 530.2519$ ).

Crystal data of penicipyrroether A (9): Colorless crystals of penicipyrroether A (9) was obtained from $\mathrm{MeOH}$. X-ray diffraction analysis was performed on an Xcalibur Atlas Gemini Ultra diffractometer (Agilent Technologies, CA, USA) with Cu K $\alpha$ radiation $(\lambda=1.54184 \AA$ ) at $100 \mathrm{~K}$. Structure was solved by direct method (SHELXL-2018) and refined with full-matrix least-squares on $F^{2}$ (ShelXL, Sheldrick, 2015). All non-hydrogen atoms were refined anisotropically and all hydrogen atoms were placed in idealized positions and refined as riding atoms with the relative isotropic parameters [2]. Crystal data of penicipyrroether $\mathrm{A}(9): \mathrm{C}_{32} \mathrm{H}_{41} \mathrm{NO}_{5}(\mathrm{M}=519.68)$, orthorhombic crystal $(0.13 \times 0.12 \times 0.09 \mathrm{~mm})$, space group P212121 (no. 19), unit cell dimensions $a=6.32760(10) \AA, b=19.8687$ (2) $\AA, c=25.0617$ (3) $\AA, V=3150.79(7) \AA^{3}, \alpha=90^{\circ}, \beta=90^{\circ}, \gamma=90^{\circ} ; Z=4 ; D_{\text {calced }}=1.231 \mathrm{~g} / \mathrm{cm}^{3} ; \mu=0.684 \mathrm{~mm}^{-1} ; 18406$ reflection measured $\left(5.676^{\circ} \leq 2 \theta \leq 147.088^{\circ}\right) ; 6237$ unique $\left(R_{\text {int }}=0.0244, R_{\text {sigma }}=0.0209\right)$ which were used in all calculation; the final refinement $(I \geq 2 \sigma(I))$ gave $R_{1}=0.0418$ and $w R_{2}=0.1116$ (all data); Flack parameter $=0.02(5)$. Crystallographic data of penicipyrroether A (9) have been deposited at the Cambridge Crystallographic Data Centre (deposition number: CCDC 1868814). Copies of the crystallographic data can be obtained free of charge via www.ccdc.cam.ac.uk/conts/retrieving.html or from the Cambridge Crystallographic Data Centre, 12, Union Road, Cambridge CB2 1EZ, U.K. [fax (+44)1223-336-033; or e-mail: data_request@ccdc.cam.ac.uk). 


\subsection{ECD Calculation}

Monte Carlo conformational searches of compound 10 were conducted with the Spartan' 10 software (v1.1.0, x 86, Wavefunction Inc., Irvine, CA, USA) using Merck Molecular Force Field (MMFF) and three conformers were obtained for ECD calculations. The X-ray CIF profile of 9 and the three conformer of 10 (Tables S3 and S4 and Figures S44 and S45) were initially optimized at B3LYP/6-31g $(\mathrm{d}, \mathrm{p})$ level in $\mathrm{MeOH}$ using the conductor-like polarizable continuum model (CPCM). The theoretical ECD calculation was carried out in $\mathrm{MeOH}$ using Time-dependent Density functional theory (TD-DFT) at the B3LYP/6-31+g $(d, p)$ level for all conformers of 9 and 10. Rotatory strengths for a total of 60 excited states for 9 (or 30 for 10) were calculated. ECD spectra were generated using the program SpecDis 1.6 (University of Würzburg, Würzburg, Germany) and GraphPad Prism 5 (University of California San Diego, USA) from dipole-length rotational strengths by applying Gaussian band shapes with sigma $=0.2 \mathrm{eV}$ for $\mathbf{9}$ (or $0.3 \mathrm{eV}$ for $\mathbf{1 0})$.

\section{6. ${ }^{13} \mathrm{C}$ NMR Calculation}

The previously described methods [19-21] were used for ${ }^{13} \mathrm{C}$ NMR calculation. Briefly, Monte Carlo conformational searches were carried out by means of Spartan's 10 software using MMFF. Four conformers (Table S8 and Figure S46) of compound 10 were obtained for NMR calculations. The conformers were initially optimized at B3LYP/6-31g $(\mathrm{d}, \mathrm{p})$ level in DMSO using the CPCM calculation model. Gauge-independent atomic orbital (GIAO) calculations of ${ }^{13} \mathrm{C}$ NMR chemical shifts were performed by DFT at the mPW1PW91-SCRF (DMSO)/6-311+g(d,p) level with the CPCM calculation model in Gaussian 09 software (G09w, D01, Gaussian Inc., Wallingford, CT, USA). The calculated ${ }^{13} \mathrm{C}$ NMR data of the lowest energy conformers for $\mathbf{1 0}$ were averaged based on the Boltzmann distribution theory and their relative Gibbs free energy.

\subsection{Sulforhodamine B (SRB) Assay}

The activity of the tested compounds in inhibiting the proliferation of human glioma U87-MG and U251 cells was determined by SRB assay [22]. Doxorubicin (DOX) was used as a positive control. U87MG cells were cultured in MEM (Minimum Essential Medium, Gibco, Grand Island, NY, USA) with 10\% FBS (Fetal Bovine Serum, PAA Laboratories Inc., Toronto, ON, Canada), U251 in DMEM (Dulbecco's Modified Eagle Medium, Gibco) and normal human astrocytes (HA) in AM (Astrocyte Medium, ScienCell, Cat. No. 1801). All cells were incubated at $37^{\circ} \mathrm{C}$ in a $5 \% \mathrm{CO}_{2}$ humidified incubator. The cultured cells after the third generation were used for experiment.

\subsection{Antibacterial Activive Assay}

The previously described micro broth dilution method [3,22] was used to evaluate the antibacterial activity of the tested compounds against the growth of MRSA and E. coli. Vancomycin (an antibiotic against MRSA) and gentamicin (an antibiotic against Gram-positive and Gram-negative bacteria) were used as positive controls. The microorganisms were cultured in MHB medium in 96-well plates at a concentration of $1 \times 10^{6} \mathrm{CFU} / \mathrm{mL}$. The MIC was determined after $12 \mathrm{~h}$ incubation at $37^{\circ} \mathrm{C}$ with tested compounds.

\section{Conclusions}

It was reported that the marine-derived Penicillium sp. ZZ380 produced a series of pyrrospirones C-I with a characteristic spiro conjunction for rings D and E in BMPM medium [2]. This study reported two new alkaloids of penicipyrroether A (9), produced by strain ZZ380 in PDB medium and pyrrospirone J (10), a previously unidentified compound isolated from a previous culture of ZZ380 in BMPM medium [2]. Both compounds 9 and 10 possess unique structures with a 6/5/6/5 fused ring system of rings $A / B / C / D$, rather than the 6/5/6/6 ring fusion for pyrrospirones $C-I$. Each of the two new alkaloids is the first compound of its structural type. Penicipyrroether A (9) is a cyclo-condensation 
product of GKK1032 analogue via the addition of a five-membered ether ring and exhibited potent inhibitory activity against the proliferation of glioma U87MG and U251 cells and the growth of MRSA and E. coli.

Supplementary Materials: The following is available online at http://www.mdpi.com/1660-3397/17/5/292/s1, Figures S1-S22: NMR, HRESIMS, UV and IR spectra of penicipyrroether A (9), Figures S23-S43: NMR, HRESIMS, UV and IR spectra of pyrrospirone J (10), Figure S44: The optimized geometry of conformer (9-1) of penicipyrroether A (9), Figure S45: The optimized geometry of conformers (10-1-10-3) of pyrrospirone J (10), Figure S46: Four conformations of the low-energy conformers of pyrrospirone J (10) calculated at B3LYP/6-31G(d) level, Table S1: ${ }^{13} \mathrm{C}$ NMR data of known compounds 11-15, Table S2: ${ }^{13} \mathrm{C}$ NMR data of known compounds 16-18, Table S3: Gibbs free energies and equilibrium populations of low-energy conformer of penicipyrroether A (9), Table S4: Cartesian coordinates for the low-energy reoptimized MMFF conformers of penicipyrroether A $(9)$ at B3LYP/6-311+G(d,p) level of theory in $\mathrm{CH}_{3} \mathrm{OH}$, Table S5: Gibbs free energies and equilibrium populations of low-energy conformers of pyrrospirone J (10), Table S6: Cartesian coordinates for the low-energy reoptimized MMFF conformers of pyrrospirone J (10) at B3LYP/6-311+G(d,p) level of theory in $\mathrm{CH}_{3} \mathrm{OH}$, Table S7: Experimental and calculated ${ }^{13} \mathrm{C}$ NMR data of pyrrospirone J (10), Table S8: Gibbs free energies and equilibrium populations of the low-energy conformers of pyrrospirone J (10) for ${ }^{13} \mathrm{C}$ NMR calculation, Table S9: Cartesian coordinates for the low-energy reoptimized MMFF conformers of pyrrospirone J (10) at B3LYP/6-311+G(d,p) level of theory in DMSO for ${ }^{13} \mathrm{C}$ NMR calculation.

Author Contributions: T.S. and M.C. conducted the isolation and culture of stain ZZ380 as well as the isolation and structural elucidation of compounds; M.T. and H.G. performed the bioactive assay; X.L. and Z.Z. designed and supervised the experiments and wrote the manuscript.

Funding: This research was supported by the National Key R\&D Program of China (No. 2017YFE0102200) and the National Natural Science Foundation of China (Nos. 81773587 and 81773769).

Conflicts of Interest: The authors declare no conflict of interest.

\section{References}

1. Li, X.W.; Ear, A.; Nay, B. Hirsutellones and beyond: Figuring out the biological and synthetic logics toward chemical complexity in fungal PKS-NRPS compounds. Nat. Prod. Rep. 2013, 30, 765-782. [CrossRef] [PubMed]

2. Song, T.F.; Chen, M.X.; Chai, W.Y.; Zhang, Z.Z.; Lian, X.Y. New bioactive pyrrospirones C-I from a marine-derived fungus Penicillium sp. ZZ380. Tetrahedron 2018, 74, 884-891. [CrossRef]

3. Song, T.; Chen, M.X.; Ge, Z.W.; Chai, W.Y.; Li, X.C.; Zhang, Z.Z.; Lian, X.Y. Bioactive penicipyrrodiether A, an adduct of GKK1032 analogue and phenol A derivative, from a marine-sourced fungus Penicillium sp. ZZ380. J. Org. Chem. 2018, 83, 13395-13401. [CrossRef]

4. Ebrahim, W.; Aly, A.H.; Wray, V.; Mándi, A.; Teiten, M.H.; Gaascht, F.; Orlikova, B.; Kassack, M.U.; Lin, W.; Diederich, M.; et al. Embellicines A and B: Absolute configuration and NF- $\mathrm{kB}$ transcriptional inhibitory activity. J. Med. Chem. 2013, 56, 2991-2999. [CrossRef] [PubMed]

5. Bode, H.B.; Bethe, B.; Höfs, R.; Zeeck, A. Big effects from small changes: Possible ways to explore nature's chemical diversity. ChemBioChem 2002, 3, 619-627. [CrossRef]

6. Harvey, A.L.; Edrada-Ebel, R.; Quinn, R.J. The re-emergence of natural products for drug discovery in the genomics era. Nat. Rev. Drug Discov. 2015, 14, 111-129. [CrossRef] [PubMed]

7. Yuan, C.; Guo, Y.H.; Wang, H.Y.; Ma, X.J.; Jiang, T.; Zhao, J.L.; Zou, Z.M.; Ding, G. Allelopathic polyketides from an endolichenic fungus Myxotrichum sp. by using OSMAC strategy. Sci. Rep. 2016, 6, 19350. [CrossRef]

8. Wang, L.; Qin, D.; Zhang, K.; Huang, Q.; Liu, S.; Han, M.J.; Dong, J.Y. Metabolites from the co-culture of nigranoic acid and Umbelopsis dimorpha SWUKD 3.1410, an endophytic fungus from Kadsura angustifolia. Nat. Prod. Res. 2017, 31, 1414-1421.

9. Xin, Z.H.; Li, T.; Zhu, T.J; Wang, W.L.; Du, L.; Fang, Y.C.; Gu, Q.Q.; Zhu, W.M. Isocoumarin derivatives from the sea squirt-derived fungus Penicillium stoloniferum QY2-10 and the halotolerant fungus Penicillium notatum B-52. Arch. Pharm. Res. 2007, 30, 816-819. [CrossRef]

10. Trisuwan, K.; Rukachaisirikul, V.; Borwornwiriyapan, K.; Phongpaichit, S.; Sakayaroj, J. Benzopyranone, benzophenone and xanthone derivatives from the soil fungus Penicillium citrinum PSU-RSPG95. Tetrahedron Lett. 2014, 55, 1336-1338. [CrossRef]

11. Cui, X.Q.; Zhu, G.L.; Liu, S.H.; Jiang, G.L.; Wang, Y.; Zhu, W.M. Diversity and function of the antarctic krill microorganisms from Euphausia superba. Sci. Rep. 2016, 6, 36496. [CrossRef] 
12. Kim, W.G.; Song, N.K.; Yoo, I.D. Quinolactacins $A_{1}$ and $A_{2}$, new acetylcholinesterase inhibitors from Penicillium citrinum. J. Antibiot. 2001, 54, 831-835. [CrossRef]

13. Shirane, N.; Takenaka, H.; Ueda, K.; Hashimoto, Y.; Katoh, K.; Ishii, H. Sterol analysis of DMI-resistant and sensitive strains of Venturia inaequalis. Phytochemistry 1996, 41, 1301-1308. [CrossRef]

14. Wakana, D.; Hosoe, T.; Itabashi, T.; Okada, K.; de Campos Takaki, G.; Yaguchi, T.; Fukushima, K.; Kawai, K. New citrinin derivatives isolated from Penicillium citrinum. J. Nat. Med. 2006, 60, 279-284. [CrossRef]

15. Lu, Z.Y.; Lin, Z.J.; Wang, W.L.; Du, L.; Zhu, T.J.; Fang, Y.C.; Gu, Q.Q.; Zhu, W.M. Citrinin dimers from the halotolerant fungus Penicillium citrinum B-57. J. Nat. Prod. 2008, 71, 543-546. [CrossRef]

16. Shiono, Y.; Shimanuki, K.; Hiramatsu, F.; Koseki, T.; Tetsuya, M.; Fujisawa, N.; Kimura, K. Pyrrospirones $\mathrm{A}$ and $\mathrm{B}$, apoptosis inducers in HL-60 cells, from an endophytic fungus, Neonectria ramulariae Wollenw KS-246. Bioorg. Med. Chem. Lett. 2008, 18, 6050-6053. [CrossRef]

17. Oikawa, H. Biosynthesis of structurally unique fungal metabolite GKK1032A2: indication of novel carbocyclic formation mechanism in polyketide biosynthesis. J. Org. Chem. 2003, 68, 3552-3557. [CrossRef]

18. Yang, X.W.; Yang, J.; Xu, G. Skeleton reassignment of type C polycyclic polyprenylated acylphloroglucinols. J. Nat. Prod. 2017, 80, 108-113. [CrossRef] [PubMed]

19. Gu, B.B.; Wu, W.; Jiao, F.R.; Jiao, W.H.; Li, L.; Sun, F.; Wang, S.P.; Yang, F.; Lin, H.W. Asperflotone, an $8(14 \rightarrow 15)$-abeo-ergostane from the sponge-derived fungus Aspergillus flocculosus 16D-1. J. Org. Chem. 2019, 84, 300-306. [CrossRef] [PubMed]

20. Cui, H.; Liu, Y.; Li, J.; Huang, X.; Yan, T.; Cao, W.; Liu, H.; Long, Y.; She, Z. Diaporindenes A-D: Four unusual 2,3-dihydro-1H-indene analogues with anti-inflammatory activities from the mangrove endophytic fungus Diaporthe sp. SYSU-HQ3. J. Org. Chem. 2018, 83, 11804-11813. [CrossRef] [PubMed]

21. Xin, W.X.; Ye, X.W.; Yu, S.R.; Lian, X.Y.; Zhang, Z.Z. New capoamycin-type antibiotics and polyene acids from marine Streptomyces fradiae PTZ0025. Mar. Drugs 2012, 10, 2388-2402. [CrossRef]

22. Ye, X.W.; Anjum, K.; Song, T.F.; Wang, W.L.; Yu, S.R.; Huang, H.C.; Lian, X.Y.; Zhang, Z.Z. A new curvularin glycoside and its cytotoxic and antibacterial analogues from marine actinomycete Pseudonocardia sp. HS7. Nat. Prod. Res. 2016, 30, 1156-1161. [CrossRef] 